\title{
Mechanism of Elementary Catalytic Steps of Pyruvate Oxidase from Lactobacillus plantarum ${ }^{\dagger}$
}

\author{
Kai Tittmann,*,‡ Ralph Golbik, ${ }^{\ddagger}$ Sandro Ghisla, ${ }^{\S}$ and Gerhard Hübner ${ }^{\ddagger}$ \\ Martin-Luther-Universität Halle-Wittenberg, Institut für Biochemie, Kurt-Mothes-Strasse 3, D-06099 Halle, Germany, and \\ Universität Konstanz, Fakultät Biologie, PF 5560-M644, D-78434 Konstanz, Germany
}

\begin{abstract}
Single steps in the catalytic cycle of pyruvate oxidase from Lactobacillus plantarum have been characterized kinetically and mechanistically by stopped-flow in combination with kinetic solvent isotope effect studies. Reversible substrate binding of pyruvate occurs with an on-rate of $6.5 \times 10^{4}$ $\mathrm{M}^{-1} \mathrm{~s}^{-1}$ and an off-rate of pyruvate of $20 \mathrm{~s}^{-1}$. Decarboxylation of the intermediate lactyl-ThDP and the reduction of FAD which consists of two consecutive single electron-transfer steps from HEThDP to FAD occur with rates of about $k_{\mathrm{dec}}=112 \mathrm{~s}^{-1}$ and $k_{\mathrm{red}}=422 \mathrm{~s}^{-1}$. Flavin radical intermediates are not observed during reduction, and kinetic solvent isotope effects are absent, indicating that electron transfer and protonation processes are not rate limiting in the overall reduction process. Reoxidation of $\mathrm{FADH}_{2}$ by $\mathrm{O}_{2}$ to yield $\mathrm{H}_{2} \mathrm{O}_{2}$ takes place at a pseudo-first-order rate of about $35 \mathrm{~s}^{-1}$ in air-saturated buffer. A comparable value of about $35 \mathrm{~s}^{-1}$ was estimated for the phosphorolysis of the acetyl-ThDP intermediate at phosphate saturation. In competition with phosphorolysis, enzyme-bound acetyl-ThDP is hydrolyzed with a rate $k$ $=0.03 \mathrm{~s}^{-1}$. This is the first report in which the reaction of enzyme-bound acetyl-ThDP with phosphate and $\mathrm{OH}^{-}$is monitored directly by FAD absorbance changes using the sequential stopped-flow technique.
\end{abstract}

Pyruvate oxidase (LpPOX $;^{1} \mathrm{EC}$ 1.2.3.3) from Lactobacillus plantarum is a homo-tetrameric flavoenzyme with a subunit molecular mass of $65.5 \mathrm{kDa}$ composed of 603 residues. Each subunit contains one tightly and noncovalently bound FAD, thiamin diphosphate (ThDP), and $\mathrm{Mg}^{2+}$ for anchoring the diphosphate moiety of ThDP. In the presence of phosphate and oxygen, $L p P O X$ catalyzes the oxidative decarboxylation of pyruvate, yielding hydrogen peroxide, carbon dioxide, and the energy storage metabolite acetyl phosphate $(1-3)$.

Catalysis of $L p$ POX can be subdivided into several steps (Scheme 1). (1) Initially the 4'-imino function of the enzymebound ThDP deprotonates C2-ThDP, forming an ylide species, which (2) attacks the carbonyl group of the substrate pyruvate in a nucleophilic manner. (3) Lactyl-ThDP decarboxylates to hydroxyethyl-ThDP, (4) which is subsequently oxidized by FAD, leading to formation of acetyl-ThDP and $\mathrm{FADH}_{2}$. (5) The reduced flavin transfers two electrons to the final acceptor $\mathrm{O}_{2}$, and $\mathrm{H}_{2} \mathrm{O}_{2}$ is released. (6) Finally, acetyl-ThDP is cleaved phosphorolytically to acetyl phosphate and ThDP.

The three-dimensional structure $(4,5)$ of $L p P O X$ has provided insight into the spatial arrangement of the enzyme-

$\dagger$ This work was supported by the Deutsche Forschungsgemeinschaft and the Fonds der Chemischen Industrie.

* Correspondence should be addressed to this author at MartinLuther-Universität Halle-Wittenberg, Institut für Biochemie, KurtMothes-Strasse 3, D-06099 Halle, Germany. Phone: 0345/5524835. Fax:0345/5527011. E-mail: kai@bc.biochemtech.uni-halle.de.

Martin-Luther-Universität Halle-Wittenberg.

$\S$ Universität Konstanz.

1 Abbreviations: ThDP, thiamin diphosphate; HEThDP, hydroxyethyl-thiamin diphosphate; $\mathrm{FADH}_{2}$, two-electron-reduced form of $\mathrm{FAD}$; $\mathrm{UV}$, ultraviolet; LpPOX, pyruvate oxidase from Lactobacillus plantarum; $E c \mathrm{POX}$, pyruvate oxidase from $E$. coli; KSIE, kinetic solvent isotope effect; ET, electron transfer; 5-dFAD, 5-carba-5-deaza-FAD. bound cofactors FAD and ThDP within the active site. However, only scattered kinetic data concerning the $L p P O X$ mechanism are available. Due to the long distance of $7 \AA$ between the two cofactors, it has been assumed (4) that the oxidation of hydroxyethyl-ThDP proceeds via two oneelectron-transfer steps.

Recent investigations have shown that the H/D exchange rate of enzyme-bound $\mathrm{C} 2-\mathrm{H}$ of ThDP is strongly affected by the presence of the second cofactor FAD and the substrate phosphate. Only in the holoenzyme complex deprotonation of the $\mathrm{C} 2-\mathrm{H}$ is sufficiently fast to account for catalysis (6, 7).

In contrast to $L p \mathrm{POX}$, the kinetic behavior of pyruvate oxidase from $E$. coli $(E c \mathrm{POX})$ has been studied in detail $(8-10)$. Activation of this enzyme by lipid binding or limited proteolysis is due to a specific enhancement of the oxidation of hydroxyethyl-ThDP by FAD. Furthermore, the ratelimiting step shifts from FAD reduction to decarboxylation through activation of the enzyme by lipid binding. Unlike $L p \mathrm{POX}$, the acetyl-ThDP intermediate in EcPOX is hydrolyzed to yield acetate. In vivo ubiquinone 8 serves as the final electron acceptor in $E c \mathrm{POX}$.

In this work we have studied the pre-steady-state and steady-state kinetics to elucidate the nature of single steps of $L p$ POX catalysis. Thus, the partial steps of decarboxylation, $\mathrm{FAD}$ reduction, $\mathrm{FADH}_{2}$ reoxidation, and phosphorolysis/hydrolysis have been characterized.

\section{EXPERIMENTAL PROCEDURES}

Chemicals and Proteins. FAD was purchased from Boehringer Mannheim; ThDP and bovine liver catalase (2000 units/mg) (EC 1.11.1.6) were from Sigma. Deuterium oxide (99.8\% enriched) was from Merck. Purified pyruvate oxidase (9 IU/mg) from Lactobacillus plantarum (EC 1.2.3.3) was 
Scheme 1

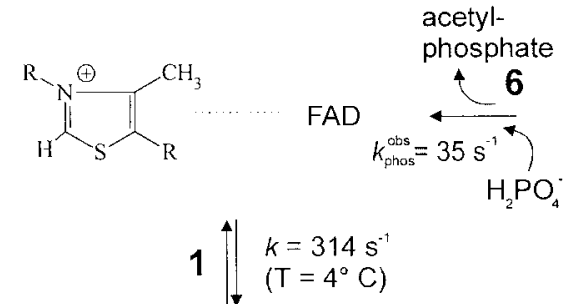<smiles></smiles>

FAD

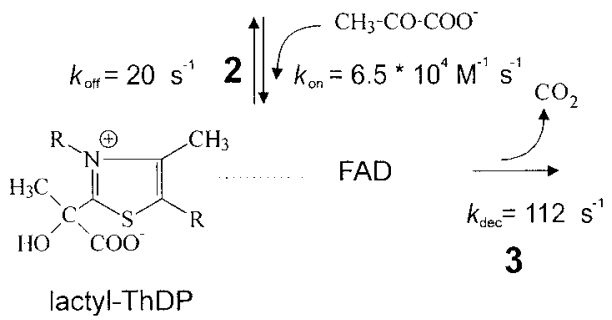

a gift from Boehringer Mannheim. Glucose oxidase (23 units/ mg) (EC 1.1.3.4) and $\beta$-D-glucose (97\%) were from Sigma. All other chemicals were of analytical grade and purchased from Merck or Boehringer Mannheim. Quartz double distilled water was used throughout the experiments.

Pretreatment of LPPOX Holoenzyme. Lyophilized pyruvate oxidase $(10 \mathrm{mg})$ was saturated with $100 \mu \mathrm{M}$ FAD, $1 \mathrm{mM}$ ThDP/ $/ \mathrm{Mn}^{2+}$ in $1 \mathrm{~mL}$ of $0.2 \mathrm{M}$ potassium phosphate buffer, $\mathrm{pH}$ 6.0, containing $20 \%(\mathrm{v} / \mathrm{v})$ glycerol, at $4{ }^{\circ} \mathrm{C}$ for $4 \mathrm{~h}$. Subsequently, excess free cofactors were removed by gel filtration on a Sephadex G25 column. The $\epsilon_{278 / 250}$ of the saturated $L p$ POX was $2.0 \pm 0.1$.

Determination of Protein Concentration and Activity Assay. Protein concentrations were determined spectrophotometrically. An extinction coefficient of $\epsilon_{278}=1.65 \mathrm{~cm}^{2} /$ mg was used for the holo-POX.

POX activity was determined via $\mathrm{H}_{2} \mathrm{O}_{2}$ production. Formation of 4-( $p$-benzoquinone-monoimino)-phenazone from the reaction of $\mathrm{H}_{2} \mathrm{O}_{2}$ with 4-aminoantipyrine $(2 \mathrm{mM})$ and 2,4-dichlorophenolsulfonic acid $(7 \mathrm{mM})$ in the presence of horseradish peroxidase $(4 \mathrm{IU} / \mathrm{mL})$ is followed spectrophotometrically at $546 \mathrm{~nm}$ with $\epsilon_{546}=16.5 \mathrm{~cm}^{2} / \mu \mathrm{mol}$. The assay buffer contained $40 \mathrm{mM}$ pyruvate in $50 \mathrm{mM}$ potassium phosphate buffer, $10 \%$ (v/v) glycerol, pH 6.0. Activity assays were performed at $25^{\circ} \mathrm{C}$.

Stopped-Flow Measurements. Rapid reaction experiments were carried out with an Applied Photophysics SX18 MV stopped-flow spectrophotometer. Holo- $L p$ POX (20 $\mu \mathrm{M}$ active site final concentration) was mixed with pyruvate $(40 \mathrm{mM}$, unless otherwise stated) in $0.2 \mathrm{M}$ potassium phosphate buffer, $\mathrm{pH} 6.0$ at $25^{\circ} \mathrm{C}$. Phosphate-free experiments were done in 0.2 M PIPES buffer, $\mathrm{pH}$ 6.0, at the same concentrations and under the same conditions. Anaerobiosis in the stopped-flow instrument was established by addition of glucose oxidase (final concentration 100 units/mL) and $20 \mathrm{mM} \beta$-D-glucose to all solutions and preincubation for $30 \mathrm{~s}$ (hermetically sealed). After this time, $L p P O X$ and varied concentrations of pyruvate were mixed as described above.<smiles>[R]c1sc(C(C)=O)[n+]([R])c1C</smiles><smiles></smiles><smiles>[R]c1sc(C(C)(O)C([O-])[OH2+])[n+]([R])c1C</smiles>

Single-wavelength measurements were carried out at 457 $\mathrm{nm}$ and with a path length of $10 \mathrm{~mm}$. A total of 1000 data points were collected for each experiment and 500 for the pre-steady-state phase $(0-0.5 \mathrm{~s})$. For sequential stopped-flow experiments, $20 \mu \mathrm{M} L p P O X$ was mixed with $80 \mathrm{mM}$ pyruvate $(1+1$ mixture) in 0.2 M PIPES buffer, $\mathrm{pH} 6.0$, in the evolving loop. After a defined delay time, $0.2 \mathrm{M}$ (unless otherwise stated) potassium phosphate buffer, $\mathrm{pH}$ 6.0, was added in a $1+10$ mixing ratio to minimize enzyme dilution. Time-resolved absorption spectra were recorded with a thermostated stopped-flow spectrophotometer equipped with a diode array detector (J\&M-Tidas) using a $10 \mathrm{~mm}$ path length cell. The wavelength range was from 300 to $700 \mathrm{~nm}$, and the acquisition time of the diode array detector was up to $0.7 \mathrm{~ms} / \mathrm{spectrum}$. All spectra are corrected for substrate and buffer absorbance.

Turnover data were analyzed according to the method of Gibson et al. (11). Kinetic traces were fitted using the programs KaleidaGraph (Synergy Software), Sigma Plot (Jandel Scientific), and Dynafit (BioKin) (12) and the appropriate algorithms. Specfit (Spectrum software associates) was used for deconvolution analyses.

Anaerobic Experiments. For anaerobic reduction of holo$L p \mathrm{POX}$, the enzyme (in $0.2 \mathrm{M}$ potassium phosphate buffer, $\mathrm{pH} 6.0$, on ice) and $5 \mathrm{mM}$ pyruvate (in a separate sidearm of the tonometer) were made anaerobic by repeated cycles of alternate evacuation and flushing with $\mathrm{O}_{2}$-free nitrogen. Absorption spectra of POX were recorded before and after anaerobiosis and during the reaction with pyruvate under anaerobic conditions with an Uvikon 930 spectrophotometer (Kontron Instruments).

\section{RESULTS AND DISCUSSION}

In the study of flavoproteins, the spectroscopic properties of the isoalloxazine system are an excellent probe for directly monitoring steps relevant to enzyme catalysis. However, this has not yet been employed to analyze single steps in the 


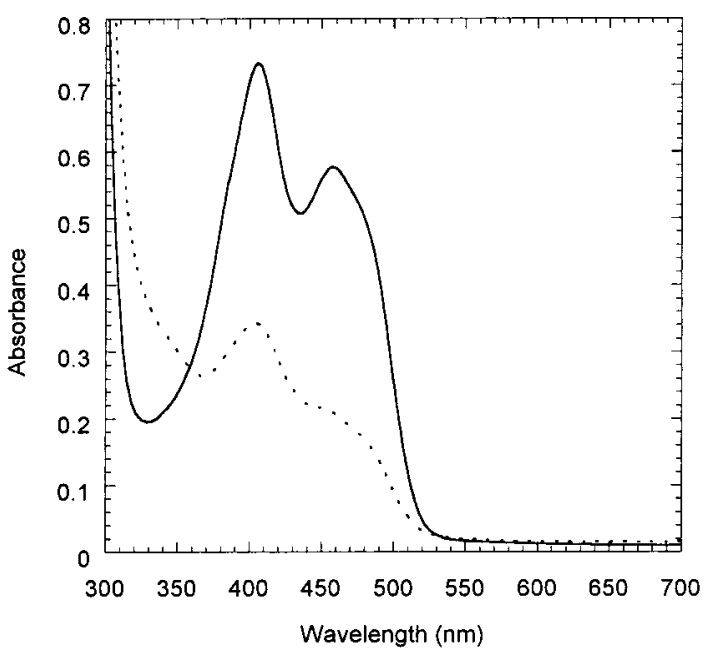

FIGURE 1: Absorption spectra of oxidized and reduced $L p$ POX. Spectra of $64 \mu \mathrm{M} L p \mathrm{POX}$ (active site concentration) in $0.2 \mathrm{M}$ potassium phosphate buffer, $\mathrm{pH}$ 6.0, were recorded before (solid line) and $60 \mathrm{~s}$ after anaerobic addition (dotted line) of $1 \mathrm{mM}$ pyruvate (final concentration) at $25^{\circ} \mathrm{C}$. The path length of the cuvette was $10 \mathrm{~mm}$. No change of the spectrum of the reduced form was observed within $60 \mathrm{~min}$, indicating no further reduction.

reaction cycle of $L p \mathrm{POX}$. The FAD fluorescence in $L p \mathrm{POX}$ has been utilized for the observation of the reconstitution of the apoprotein with the cofactor and for monitoring association equilibria and stability $(6,13)$.

The two lowest $\pi-\pi^{*}$ transitions of $L p$ POX-bound oxidized FAD are at 408 and $457 \mathrm{~nm}$. The intensity ratio of these two bands in $L p P O X$ is inverted with respect to free FAD and is reminiscent of that for cholesterol oxidase (14) and oxynitrilase (15). Upon anaerobic reduction of the enzyme by its substrate pyruvate, the enzyme-bound reduced flavin exhibits an absorption spectrum with $\lambda_{\max }$ at 400 and $450 \mathrm{~nm}$. No absorbance indicative for enzyme-bound flavosemiquinone species is observed in this type of experiment (Figure 1).

Course of FAD Reduction during LPPOX Catalysis in the Presence of Phosphate. To investigate the reductive halfreaction of the enzyme-bound FAD during $L p P O X$ catalysis, the conversion was initiated by mixing the protein solution with the substrate pyruvate in air-saturated potassium phosphate buffer $(0.2 \mathrm{M}, \mathrm{pH} 6.0)$. The absorbance changes were monitored with the stopped-flow instrument at $\lambda=457$ $\mathrm{nm}$. As shown in Figure 2, the course of enzyme-bound FAD reduction during $L p P O X$ catalysis consists of several phases. In the pre-steady-state phase, an initial decrease in absorbance occurs within $20 \mathrm{~ms}$ and is followed by a small, but significant increase (Figure 2, inset). The decrease in absorbance showing a distinct lag phase reflects mainly the reduction of FAD by the intermediate HEThDP and all preceding steps, such as binding of pyruvate and decarboxylation of the lactyl-ThDP intermediate. The following small increase in absorbance can be expected in the case where reoxidation of the reduced FAD by oxygen is followed by an additional slow step before the oxidized enzyme is generated. This additional step should be the phosphorolysis of the acetyl-ThDP intermediate to yield acetyl phosphate. A minimal mechanism in accordance with the experimental data is shown in Scheme 1. After the pre-steady-state phase, the steady-state between reduced and oxidized enzyme-FAD species is established.

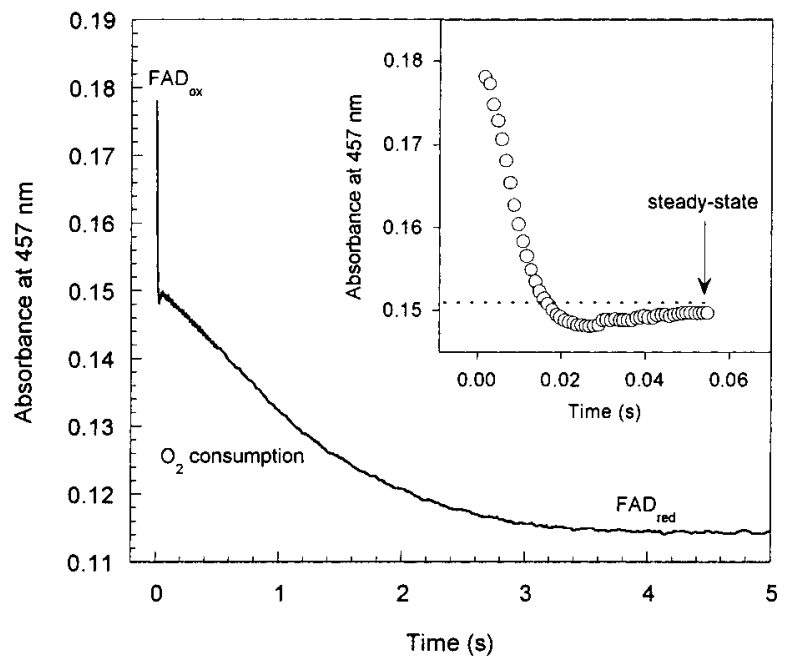

FIGURE 2: Course of $L p P O X$ reaction with pyruvate at $457 \mathrm{~nm}$ starting from oxidized enzyme. A solution of $20 \mu \mathrm{M}$ oxidized LpPOX (active site concentration) in $0.2 \mathrm{M}$ potassium phosphate buffer, $\mathrm{pH} 6.0$ (air saturation), was reacted with $20 \mathrm{mM}$ pyruvate at $25{ }^{\circ} \mathrm{C}$ in the stopped-flow instrument (optical path length 10 $\mathrm{mm})$. Inset: Initial phase of the reaction $(0-60 \mathrm{~ms})$.

Scheme 2

$$
\begin{aligned}
& \mathrm{E}_{\text {ThDP }}^{\prime}{ }_{\mathrm{TAD}}^{\mathrm{C}}+\mathrm{CH}_{3} \mathrm{COCOO} \underset{k_{\text {off }}}{\stackrel{k_{\text {on }}}{\longrightarrow}} \mathrm{E}_{\text {Lactyl-ThDP }}^{\prime} \stackrel{\text { FAD }}{\stackrel{k_{\text {dec }}}{\longrightarrow}}
\end{aligned}
$$

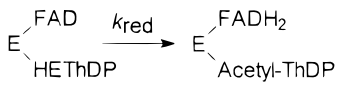

During turnover the oxygen concentration then decreases and, in the presence of excess pyruvate, the steady-state ratio of $\mathrm{FAD} / \mathrm{FADH}_{2}$ shifts until the $L p \mathrm{POX}-\mathrm{FAD}$ is completely reduced (Figure 2).

Back electron transfer from $\mathrm{FADH}_{2}$ to acetyl-ThDP is negligible, consistent with the equilibrium being shifted largely to the right (data not shown). This follows from the observation that in the absence of oxygen pyruvate completely reduces enzyme-bound FAD to $\mathrm{FADH}_{2}$.

Reductive Half-Reaction. To investigate steps of the reductive half-reaction (Scheme 2), we started the reaction with pyruvate after the complete removal of oxygen in the reaction mixture by addition of glucose oxidase and $\beta$-Dglucose. In these single-turnover experiments, the progress curves of the reduction of FAD (Figure 3) show lag phases as already observed in the pre-steady-state in the presence of oxygen (Figure 2). The time course of the decrease in FAD absorbance is determined by several factors. The observation of a lag phase, which is independent of the concentration of the substrate pyruvate at saturation and that precedes FAD reduction, indicates that the step following substrate binding (step 2, Scheme 1), i.e., decarboxylation (step 3, Scheme 1), can be separated from the subsequent reduction of the flavin (step 4, Scheme 1). The best fit of the FAD reduction (Figure 3) according to Scheme 2 using the program Dynafit yields rates of $k_{\text {on }}=(6.5 \pm 0.2) \times 10^{4}$ $\mathrm{M}^{-1} \mathrm{~s}^{-1}$ and $k_{\mathrm{off}}=20 \pm 3 \mathrm{~s}^{-1}$ for the reversible substrate binding of pyruvate, a value of $k_{\mathrm{dec}}=112 \pm 20 \mathrm{~s}^{-1}$ for the decarboxylation of lactyl-ThDP, and a value of $k_{\text {red }}=422$ $\pm 14 \mathrm{~s}^{-1}$ for the reduction step of FAD, respectively. Interestingly, the rates of substrate binding and the off-rate from the enzyme, as well as the rate of the decarboxylation, are in the same range as found for the enzyme pyruvate 


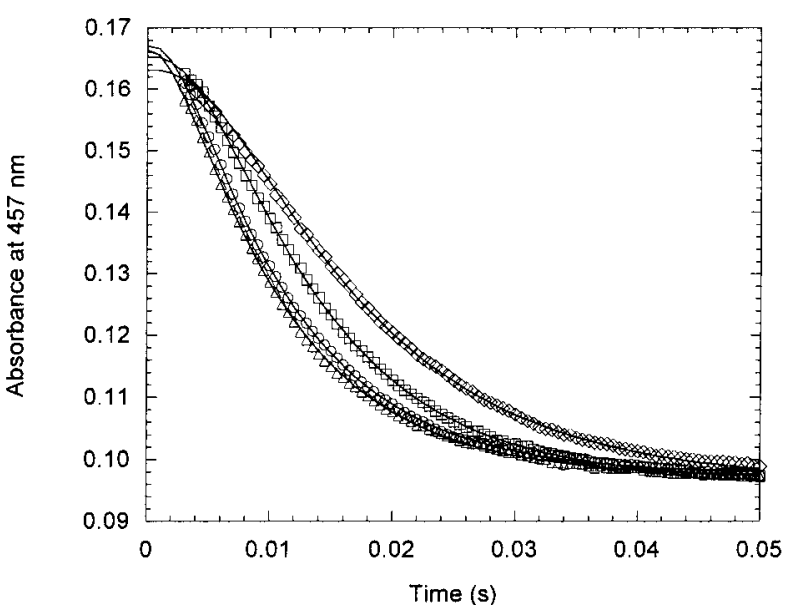

FIGURE 3: Reaction of oxidized pyruvate oxidase with pyruvate in the absence of oxygen. The reaction of $20 \mu \mathrm{M} L p$ POX (active site concentration) in $0.2 \mathrm{M}$ oxygen-free potassium phosphate buffer, pH 6.0, with $2.5 \mathrm{mM}(\diamond), 5 \mathrm{mM}(\square), 20 \mathrm{mM}(\bigcirc)$, and $50 \mathrm{mM}$ pyruvate $(\triangle)$, respectively, at $25^{\circ} \mathrm{C}$ was followed with a stoppedflow instrument with an optical path length of $10 \mathrm{~mm}$. The fit of the data points (full lines) based on Scheme 2 using the program Dynafit yields rate constants of reversible substrate binding $k_{\text {on }}=$ $6.5 \times 10^{4} \mathrm{M}^{-1} \mathrm{~s}^{-1}$ and $k_{\mathrm{off}}=20 \mathrm{~s}^{-1}$, of decarboxylation $k_{\mathrm{dec}}=$ $112 \mathrm{~s}^{-1}$, and of reduction $k_{\mathrm{red}}=422 \mathrm{~s}^{-1}$, respectively.

decarboxylase (16). The assignment of rate constants to irreversible steps (decarboxylation, FAD reduction) of the reductive half-reaction is nonambiguous at subsaturation of pyruvate as simulated with Dynafit (data not shown). Further results to support the proposed assignment are presented below under Catalysis in the Presence of 2,6-Dichlorophenolindophenol.

Catalysis Starting from Reduced LPPOX. When catalysis is started by addition of air-saturated buffer to completely reduced $L p \mathrm{POX}$, steady-state is approached at a rate $k_{\mathrm{obs}}=$ $70 \pm 6 \mathrm{~s}^{-1}$ (Figure 4) that is significantly slower than the rate of the initial decrease obtained starting from the oxidized LpPOX-FAD and pyruvate but is comparable with the rate of the following small increase in absorbance in this experiment (compare with inset in Figure 2). Considering that this $k_{\text {obs }}$ reflects the total of $k_{\text {reox }}$ and $k_{\text {phos }}$, this indicates that decarboxylation (step 3) as well as the reduction of FAD by HEThDP (step 4) occurs at a significantly higher rate than reoxidation (step 5) and phosphorolysis (step 6).

Steady-State Ratio of Oxidized and Reduced Enzyme Species. At pyruvate saturation, the concentration ratio of FAD and $\mathrm{FADH}_{2}$ in the steady-state is dependent on $k_{\text {dec }}$ (step 3), $k_{\text {red }}$ (step 4), and on the rates of the reoxidation by oxygen, $k_{\text {reox }}$ (step 5), and of phosphorolysis of the acetylThDP intermediate, $k_{\text {phos }}$ (step 6). This situation can be described by eq 1 :

$$
\frac{[\mathrm{FAD}]}{\left[\mathrm{FADH}_{2}\right]}=\frac{k_{\mathrm{reox}}}{k_{\mathrm{dec}}}+\frac{k_{\mathrm{reox}}}{k_{\mathrm{red}}}+\frac{k_{\mathrm{reox}}}{k_{\mathrm{phos}}}
$$

A concentration ratio $[\mathrm{FAD}] /\left[\mathrm{FADH}_{2}\right] \approx 1.3$ can be estimated from Figure 2 at steady-state. Taking this value together with the rates of decarboxylation and reduction and the total of rates of reoxidation and phosphorolysis $\left(k_{\mathrm{obs}}=\right.$ $\left.70 \pm 6 \mathrm{~s}^{-1}\right)$, comparable values of $k_{\text {reox }}$ and of $k_{\text {phos }}$ of about $35 \pm 6 \mathrm{~s}^{-1}$ can be calculated. This clearly indicates that the rate-limiting steps in $L p P O X$ catalysis are the reoxidation

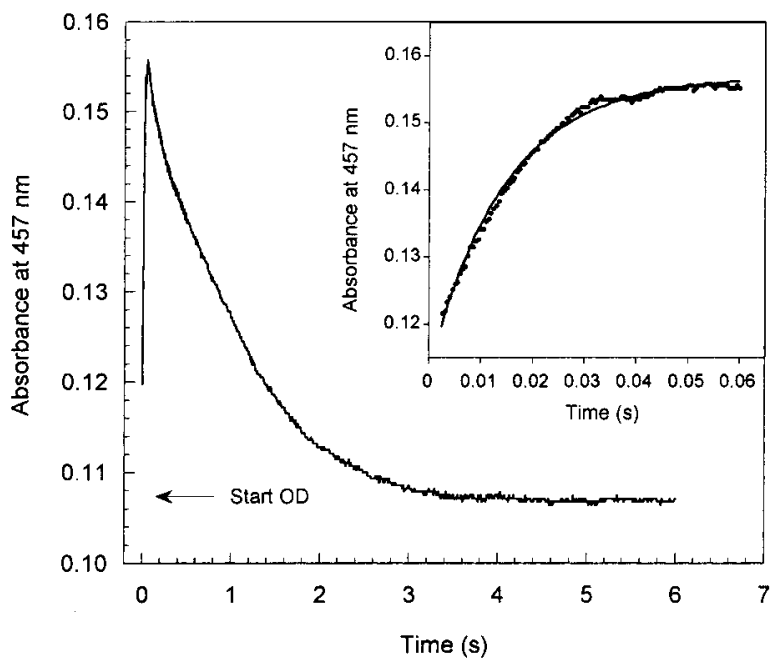

FIGURE 4: Course of the $L p P O X$ reaction with pyruvate at $457 \mathrm{~nm}$ starting from reduced enzyme. A solution of $240 \mu \mathrm{M} L p \mathrm{POX}$ (active site concentration) was mixed with $40 \mathrm{mM}$ pyruvate in $0.2 \mathrm{M}$ potassium phosphate buffer, $\mathrm{pH} 6.0$ (air saturated), in a $1+1$ mixing ratio. The reaction was allowed to proceed until completion of flavin reduction in the sequential flow evolving loop of the stopped-flow instrument (see Figure 2). At this time point, $0.2 \mathrm{M}$ air-saturated potassium phosphate buffer, $\mathrm{pH} 6.0$, containing $20 \mathrm{mM}$ pyruvate was added in a 5+1 mixing ratio, and the reaction course was monitored at $\lambda=457 \mathrm{~nm}$ and $25{ }^{\circ} \mathrm{C}$. Inset: Initial phase. The increase in absorbance is fitted (full line) to a single exponential function with a rate constant $k_{\mathrm{obs}}=70 \mathrm{~s}^{-1}\left(k_{\mathrm{obs}}=k_{\text {reox }}+k_{\mathrm{phos}}\right)$.

by oxygen and the phosphorolysis of the acetyl-ThDP intermediate.

The area delimited by the kinetic trace of the steady-state phase depicted in Figure 2 corresponds to the initial oxygen concentration of $253 \mu \mathrm{M}$. Using the method of Gibson (11), a $k_{\text {cat }}=16 \pm 2 \mathrm{~s}^{-1}$ for $L p$ POX catalysis can be estimated, which is in good agreement with the value calculated from the rate constants of the individual steps according to eq 2 $\left(k_{\mathrm{cat}}=14.6 \mathrm{~s}^{-1}\right)$ :

$$
\begin{aligned}
& k_{\text {cat }}= \\
& \frac{k_{\text {dec }} k_{\text {red }} k_{\text {reox }} k_{\text {phos }}}{k_{\text {red }} k_{\text {reox }} k_{\text {phos }}+k_{\text {reox }} k_{\text {phos }} k_{\text {dec }}+k_{\text {phos }} k_{\text {dec }} k_{\text {red }}+k_{\text {dec }} k_{\text {red }} k_{\text {reox }}}
\end{aligned}
$$

Detection of Reaction Intermediates by Time-Resolved Rapid Spectroscopy. Since no flavosemiquinone species were detectable in static experiments such as those of Figure 1, we used time-resolved rapid spectroscopy to ascertain the transient occurrence of radicals during the reduction of FAD by HEThDP. As shown in Figure 5, neither blue nor red flavosemiquinone species can be detected in the early phases of the reaction. The flavin absorbance appears to be composed only of oxidized and fully reduced flavin species (Figure 5).

Kinetic Solvent Isotope Effect. To address the question, whether the reduction in $L p P O X$ proceeds in a two-step single electron transfer or possibly via a hydride transfer after protonation of the carbanion of HEThDP, kinetic solvent isotope effect (KSIE) experiments were performed. Singlewavelength $(\lambda=457 \mathrm{~nm})$ stopped-flow experiments were carried out with pyruvate and phosphate at saturating concentrations and in air-saturated buffer using different mole fractions of deuterium oxide at $\mathrm{pL} 6.0[\mathrm{pH}$-meter readings 


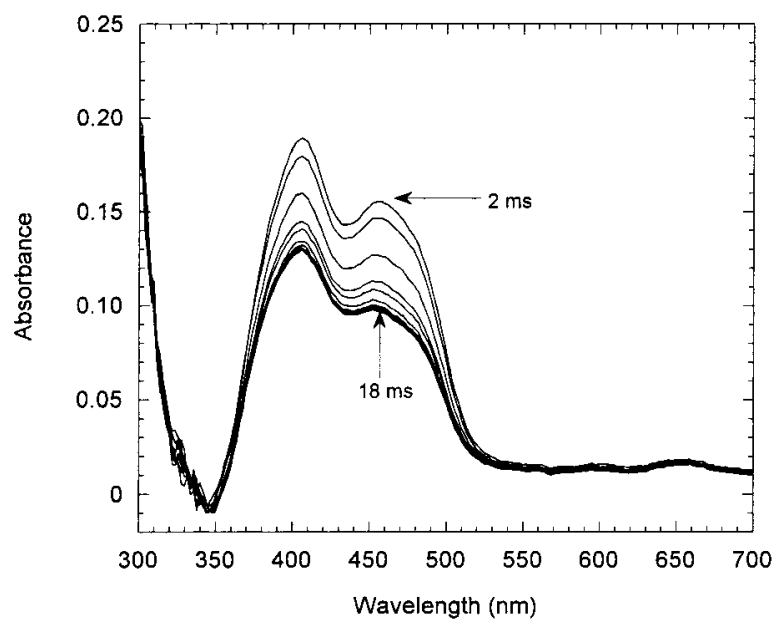

FIGURE 5: Time-resolved absorption spectra of $L p$ POX-bound FAD during approach of the steady-state and in the presence of phosphate. The absorption spectra of enzyme-bound FAD were recorded after mixing a solution of $40 \mu \mathrm{M} L p P O X$ (active sites) and $40 \mathrm{mM}$ pyruvate in a $1+1$ mixing ratio in $0.2 \mathrm{M}$ potassium phosphate buffer, pH 6.0 at $25^{\circ} \mathrm{C}$ (optical path length $10 \mathrm{~mm}$ ). The total acquisition time was $18 \mathrm{~ms}$, and spectra recorded at increments of $2 \mathrm{~ms}$ are shown. The spectra are corrected for contributions of buffer and substrate.

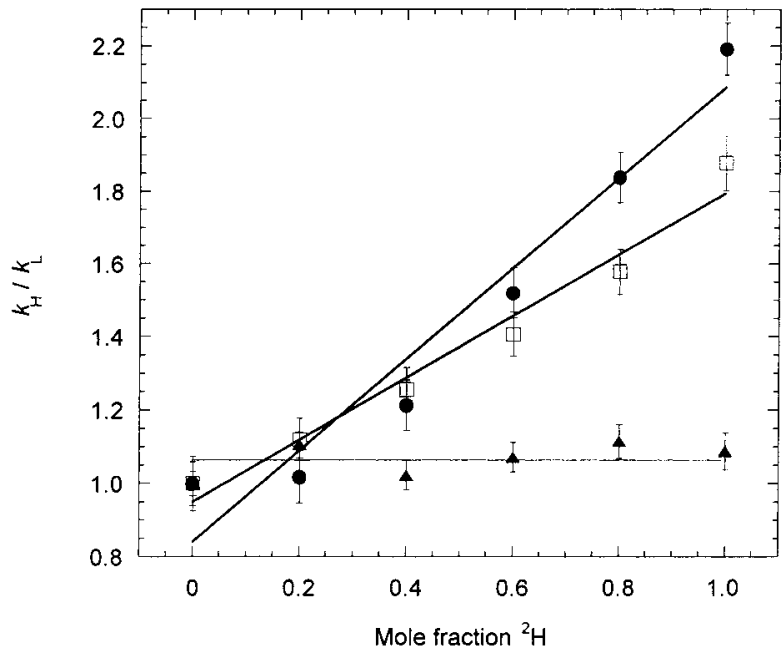

FIGURE 6: Proton inventory for single steps of $L p$ POX catalysis. The rate constants of the reduction $(\boldsymbol{\Lambda})$ and the $k_{\text {cat }}$ determined from the area under the kinetic trace of the stopped-flow measurements ( $\square$ ) and from $\mathrm{H}_{2} \mathrm{O}_{2}$ formation ( $)$ were determined in different molar fractions of $\mathrm{H}_{2} \mathrm{O}$ and $\mathrm{D}_{2} \mathrm{O}$. The standard assay contained 20 $\mu \mathrm{M} L p \mathrm{POX}$ (active site concentration) and $20 \mathrm{mM}$ pyruvate in airsaturated potassium phosphate buffer $(0.2 \mathrm{M}, \mathrm{pH}$ 6.0). The rate constants obtained in $\mathrm{H}_{2} \mathrm{O}$ buffer $\left(k_{\mathrm{H}}\right)$ were divided by the rate constants of the reaction in $\mathrm{L}_{2} \mathrm{O}$ buffer $\left(k_{\mathrm{L}}\right)$.

were corrected according to (32), p 244]. Lyophilized $L p P O X$ and pyruvate were dissolved in mixtures of air-saturated potassium phosphate buffer. To differentiate between transfer intrinsic and effects due to $\mathrm{H} / \mathrm{D}$ exchange processes on the protein, $L p \mathrm{POX}$ and pyruvate were reacted immediately after dissolving in the preequilibrated buffer. No kinetic solvent isotope effect was found (Figure 6). However, the ratelimiting step(s) of $L p \mathrm{POX}\left(k_{\text {cat }}\right)$ catalysis show(s) a KSIE of $k_{\text {cat }}\left(\mathrm{H}_{2} \mathrm{O}\right) / k_{\text {cat }}\left(\mathrm{D}_{2} \mathrm{O}\right) \approx 1.8 \pm 0.2$ and a linear dependence of the proton inventory curve measured under both stoppedflow and steady-state conditions by the $\mathrm{H}_{2} \mathrm{O}_{2}$-detecting assay (Figure 6).

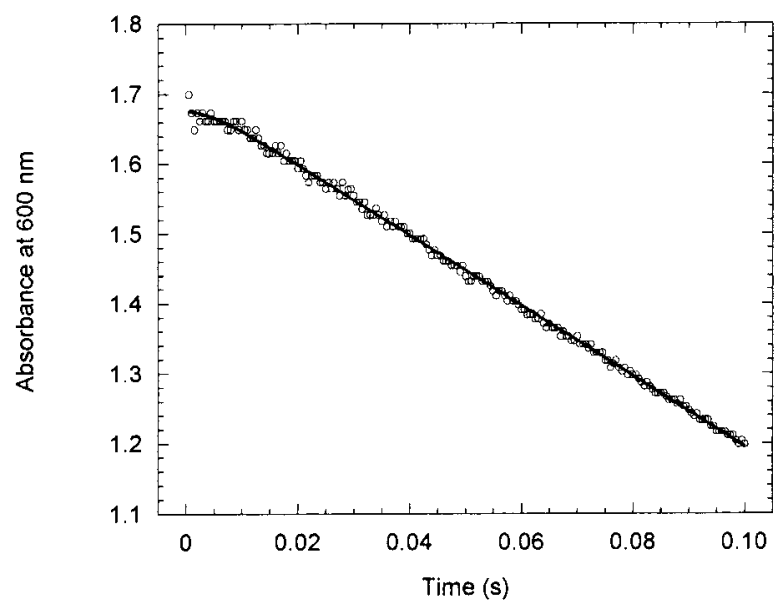

FIGURE 7: Time course of $L p P O X$ reaction in the presence of the artificial electron acceptor 2,6-dichlorophenolindophenol. The reaction of $20 \mu \mathrm{M} L p \mathrm{POX}$ (active site concentration) in $0.2 \mathrm{M}$ potassium phosphate buffer, $\mathrm{pH} 6.0$, with $20 \mathrm{mM}$ pyruvate and $130 \mu \mathrm{M} 2,6-$ dichlorophenolindophenol at $25^{\circ} \mathrm{C}$ was monitored at $600 \mathrm{~nm}$ using the stopped-flow instrument. The line represents a fit according to a single exponential first-order reaction with a rate constant of $k_{\text {lag }}$ $=185 \pm 40 \mathrm{~s}^{-1}$ including a linear term: $A_{600}(t)=A_{0}{ }^{\mathrm{ex}}-\Delta A / \Delta t_{\mathrm{SS}}$ $-A_{\mathrm{x}} \cdot \exp \left(-k_{\mathrm{lag}} t\right)$, where $A_{0}{ }^{\mathrm{ex}}$ is the extrapolated absorbance at $t=$ $0, \Delta A / \Delta t_{\mathrm{SS}}$ is the rate at steady-state $\left(\mathrm{s}^{-1}\right), A_{\mathrm{x}}$ is the amplitude of the lag phase, and $k_{\text {lag }}$ is the rate constant of the lag phase $\left(\mathrm{s}^{-1}\right)$.

To test whether protein H/D exchange affects the rates of reduction and of $k_{\text {cat }}$, the protein was preincubated in solvent isotope mixtures for definite time intervals. Since $L p P O X$ is prone to aggregation in pure deuterium oxide, the experiments were done in 0.6 mole fraction of deuterium oxide. In comparison to the results presented in Figure 6, and for incubation times of up to $5 \mathrm{~h}$, the same rate constants for the single steps and for $k_{\text {cat }}$ were found (data not shown). Therefore, we conclude that protein H/D exchange does not affect the measured rates.

Catalysis in the Presence of 2,6-Dichlorophenolindophenol. In the presence of the artificial electron acceptor 2,6dichlorophenolindophenol and of phosphate, $L p$ POX catalyzes the oxidative decarboxylation of pyruvate, yielding acetyl phosphate also in the absence of oxygen (6). A lag phase is observed in the progress of this reaction, monitored at $600 \mathrm{~nm}$, before the steady-state is established (Figure 7). The lag phase corresponds to a step with a rate constant $k=$ $185 \pm 40 \mathrm{~s}^{-1}$, whereas from the steady-state a catalytic constant of $k_{\mathrm{cat}}=26 \mathrm{~s}^{-1}$ was determined using an extinction coefficient of $\epsilon_{600}=12.7 \mathrm{~cm}^{2} / \mu \mathrm{mol}$.

In this artificial reaction 2,6-dichlorophenolindophenol reacts with the carbanion of the enzyme-bound intermediate HEThDP (6). Therefore, the lag phase can be attributed to the decarboxylation reaction. The estimated value is in the same range as determined in the native reaction by detecting the FAD reduction and provides further evidence for the correct assignment of the individual steps in the reductive half-reaction (Figure 3 ). The cause of the higher $k_{\text {cat }}$ of $L p \mathrm{POX}$ in the presence of 2,6-dichlorophenolindophenol is described in (6).

FAD Reduction in the Absence of Phosphate. In phosphatefree solution (0.1 M PIPES buffer, $\mathrm{pH}$ 6.0), the reaction of $L p$ POX with pyruvate (monitored at $\lambda=457 \mathrm{~nm}$ ) consists of several phases (Figure 8). At first, enzyme-bound FAD is rapidly reduced as already observed in the presence of 


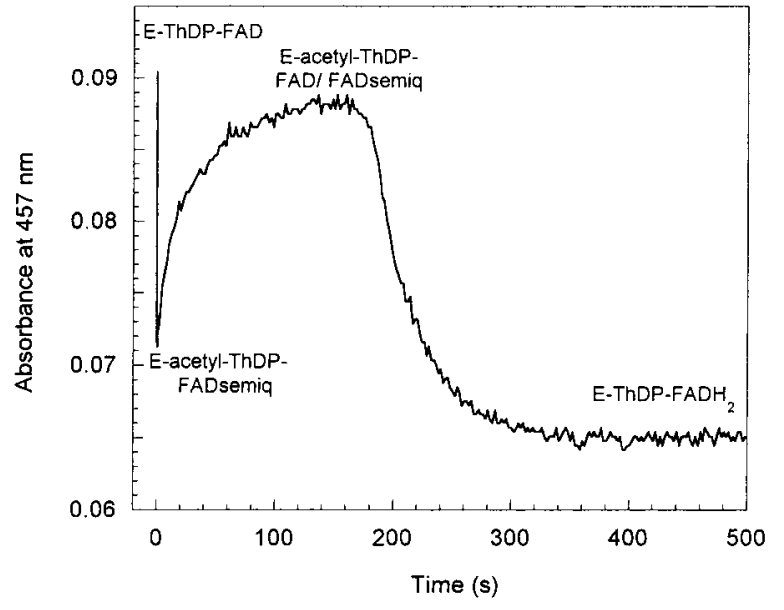

FIGURE 8: Progress curve of $L p P O X$ reaction in the absence of phosphate monitored at $457 \mathrm{~nm}$. Reaction of $10 \mu \mathrm{M} L p \mathrm{POX}$ (active site concentration) in 0.1 M PIPES buffer, $\mathrm{pH} 6.0$ (air saturated), with $20 \mathrm{mM}$ pyruvate (final concentrations) at $25^{\circ} \mathrm{C}$ was monitored using a stopped-flow instrument. The intermediates are inscribed within the progress curve.

phosphate. This reduction is followed, up to $\approx 150 \mathrm{~s}$, by a slower return of absorbance. A further decrease in absorbance then ensues as a consequence of oxygen consumption. To characterize intermediates formed in the pre-steady-state, this phase was investigated by time-resolved spectroscopy (Figure 9A). The initial fast decrease in absorbance at $457 \mathrm{~nm}$ is accompanied by an increase at $\lambda>500 \mathrm{~nm}$ and a shift of the $\lambda_{\max }$ in the $350-400 \mathrm{~nm}$ region.

These species may be interpreted as being composed of oxidized and half-reduced (semiquinone) FAD. However, the spectra do not suggest the exclusive presence of either the red or the blue semiquinone. Thus, we assume that both the neutral and the anionic species are present, the second being the predominant form. A high amount of a species containing oxidized FAD having a strong absorption at $380 \mathrm{~nm}$ (Figure 9B) is accumulated slowly with a rate constant of $k_{\mathrm{obs}}=$ $0.03 \mathrm{~s}^{-1}$ in the pre-steady-state, indicating that in this case the regeneration of the free enzyme is limited by a very slow step. The rate constant of the relaxation process should reflect the hydrolysis of the intermediate acetyl-ThDP.

The kinetic course of the pre-steady-state was studied by sequential stopped-flow photometry starting the reaction from the oxidized intermediate (accumulated in phosphate-free solution); experiments with single-wavelength detection at $\lambda=457 \mathrm{~nm}$ were performed. The enzyme was premixed with pyruvate (both in $0.1 \mathrm{M}$ PIPES buffer, $\mathrm{pH}$ 6.0) in the evolving loop until the reoxidation process had finished. At this point in time, the solution was reacted with a 10 -fold excess of phosphate $(0.2 \mathrm{M}$ potassium phosphate buffer, $\mathrm{pH}$ 6.0). The rate of the ensuing process can be estimated as $k_{\mathrm{obs}}=7.9 \mathrm{~s}^{-1}$ (Figure 10). The correlation of this rate to specific steps in catalysis is difficult, because it is suggested that phosphorolysis of acetyl-ThDP starts from a different intermediate in comparison to the reaction starting with pyruvate in phosphate buffer (Figure 2, compare also Figure 5 and Figure 9B).

\section{DISCUSSION OF SINGLE STEPS}

Decarboxylation. The low limit of the rate constant of step 3 (Scheme 1) preceding flavin reduction (step 4) can be
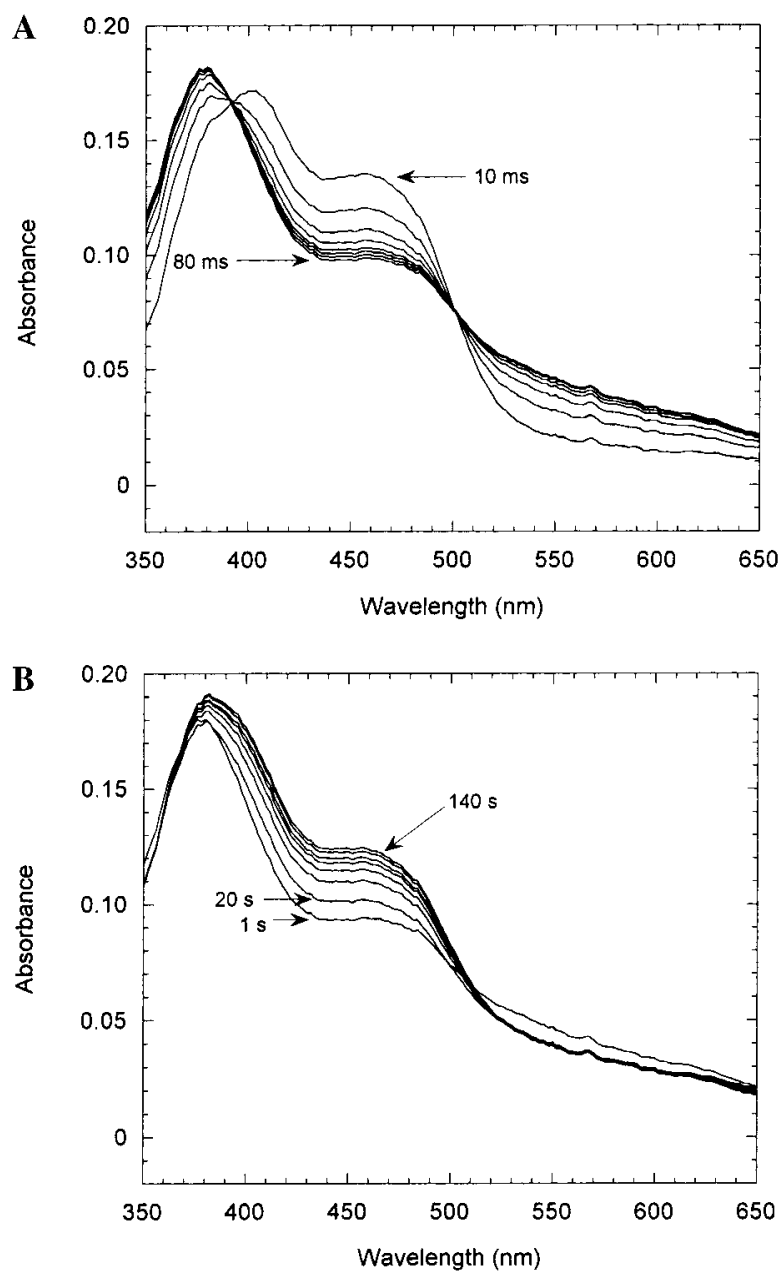

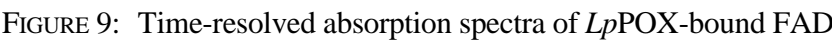
during the first turnover reduction in the absence of phosphate. The absorption spectra of enzyme-bound FAD between 300 and 700 $\mathrm{nm}$ were recorded after mixing $20 \mu \mathrm{M} L p \mathrm{POX}$ (active site concentration) and $20 \mathrm{mM}$ pyruvate (final concentrations) in 0.1 $\mathrm{M}$ PIPES buffer, $\mathrm{pH} 6.0$ at $25^{\circ} \mathrm{C}$. The total acquisition time was $80 \mathrm{~ms}$ (A) and $140 \mathrm{~s} \mathrm{(B);} \mathrm{the} \mathrm{time} \mathrm{increment} \mathrm{was} \mathrm{set} \mathrm{to} 10 \mathrm{~ms}$ (A) and $20 \mathrm{~s}$ (B), respectively. All spectra are corrected for buffer and substrate contributions.

estimated as $k_{\mathrm{dec}}=112 \pm 20 \mathrm{~s}^{-1}$ from the extent of the preceding lag phase (Figure 3). In comparison with the rates of the reoxidation and phosphorolysis, the decarboxylation is not rate-limiting in $L p P O X$ catalysis, in contrast to activated $E c \mathrm{POX}$ (8) or to pyruvate decarboxylase, which show a partial rate-limiting decarboxylation (18). For comparison, the decarboxylation of free lactyl-ThDP is about $4 \times 10^{-4} \mathrm{~s}^{-1}$ (19). Thus, the acceleration induced by the enzyme is approximately 6 orders of magnitude. Lienhard suggested that this acceleration may result from a nonpolar microenvironment within the active site or from the involvement of a Brønsted acid capable of protonating the $\alpha$-carbanion after decarboxylation $(20,21)$. In $L p P O X$, we suggest that the apolar residues Phe479 and Val394, which are in contact with the ThDP, are responsible for the effect. In addition, these residues might affect the stereospecific orientation of the carboxylate of enzyme-bound lactyl-ThDP, perpendicular to the plane of the thiazolium ring (22). In LpPOX, protonation of the $\alpha$-carbanion clearly cannot be the force driving the reaction as suggested in (24).

Reduction of FAD. The reduction of enzyme-bound FAD by the HEThDP intermediate proceeds via a two-step single- 


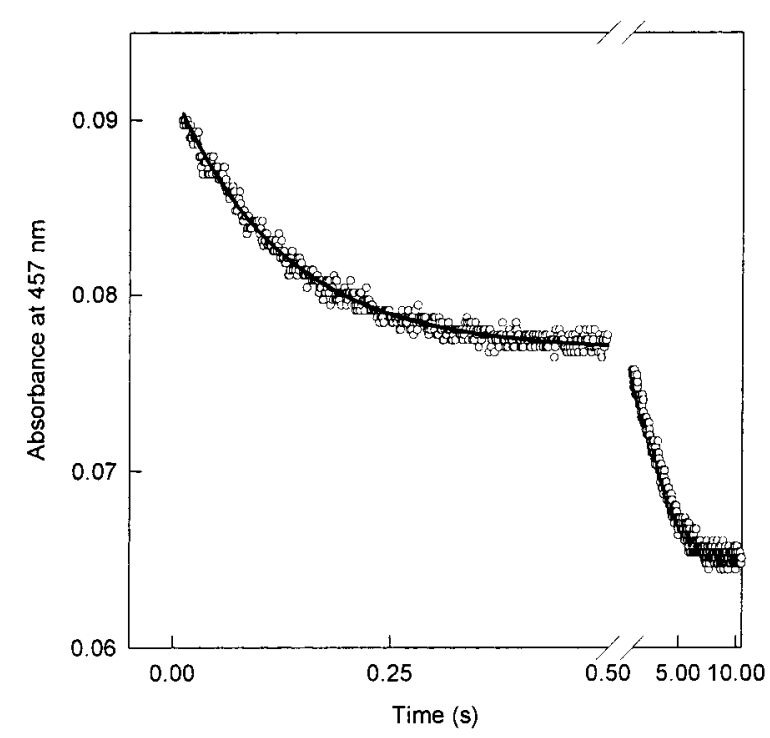

FIGURE 10: Phosphorolysis-limited turnover of $L p$ POX. $20 \mu \mathrm{M}$ $L p \mathrm{POX}$ (active site concentration) was mixed with $20 \mathrm{mM}$ pyruvate in $0.1 \mathrm{M}$ PIPES buffer, $\mathrm{pH} 6.0$, in a $1+1$ mixing ratio and the reaction permitted in the sequential flow evolving loop of the stopped-flow instrument until the acetyl-ThDP-FAD-POX intermediate was formed (see Figure 8). At this time point, $200 \mathrm{mM}$ phosphate was added in a $1+10$ ratio, and the reduction of enzymebound FAD was recorded at $\lambda=457 \mathrm{~nm}$ and $25^{\circ} \mathrm{C}$. The kinetic trace of the first phase was fitted to a single exponential first-order reaction with $k_{\text {phos }}=7.9 \mathrm{~s}^{-1}$.

electron-transfer mechanism with $k_{\mathrm{obs}}=422 \pm 14 \mathrm{~s}^{-1}$ at saturating concentrations for both pyruvate and phosphate. A mechanism that involves protonation of the carbanion of HEThDP and subsequent hydride transfer, which has been proposed for several flavin-dependent dehydrogenases (23), is unlikely since no KSIE is evident for this catalytic step (Figure 5). In line with this, when FAD in $L p \mathrm{POX}$ is replaced by $5 \mathrm{~d}-\mathrm{FAD}$, no reduction is observed by the HEThDP intermediate (5d-flavins do not catalyze transfer of single electrons; they function as hydride acceptors) (6).

A direct $\alpha$-carbanion mechanism with formation of a covalent adduct is very unlikely due to the steric orientation of the two cofactors and the long distance between ThDP and FAD. The distance between the $\mathrm{C} 2$ of the ThDP and the $\mathrm{C} 4 \mathrm{a}$ of the FAD is about $11 \AA$ as derived from the $\mathrm{X}$-ray crystallography structure $(4,5)$. Our results are in good agreement with the findings of Jordan and co-workers (23), who have demonstrated that $\mathrm{C}_{\alpha}$-deprotonated HEThDP reduces a flavin analogue in a two-step electron-transfer reaction in solution. Moreover, Nakanishi et al. $(25,26)$ conclude from the electron-transfer properties of free HEThDP that it may act as a two-electron donor with potentials of $E_{\mathrm{ox}(1)}^{0}=-0.97 \mathrm{~V}$ and $E_{\mathrm{ox}(2)}^{0}=-0.56 \mathrm{~V}$.

Despite these clear indications for a radical two-step transfer mechanism, no populated flavin radical could be detected, neither during the first-turnover electron transfer (Figure 5) nor under steady-state conditions or upon anaerobic reduction (Figure 1). It is unclear by which pathway the electrons are transferred between HEThDP and FAD. Based on the X-ray crystallography structure, Muller and coworkers $(4,5)$ have assumed that a stepwise electron transfer may be relayed by the benzene rings of Phe 479 and/or Phe121 of the other subunit. On the other hand, the electrons may simply tunnel from HEThDP to FAD as proposed by
Dutton and co-workers (27). Since the redox potentials of the $L p$ POX-bound HEThDP and of the FAD are still unknown, it is difficult to calculate the theoretical rate of electron transfer (ET) from HEThDP to FAD according to (27). From the X-ray structure, the edge-to-edge distance can be estimated as $6.5 \AA$ and the packing density between both cofactors as $\rho=0.47$. Assuming a $\Delta \mathrm{G}=0 \mathrm{eV}$ and a reorganization energy of $0.7 \mathrm{eV}$, the theoretical rate of ET exceeds by far the observed rate of reduction $\left(k_{\text {theo }} \approx 10^{8}\right.$ $\left.\mathrm{s}^{-1}, k_{\mathrm{obs}}=422 \mathrm{~s}^{-1}\right)$. Thus, it is very unlikely that the rate of reduction reflects the intrinsic rate of electron transfer. The absence of a KSIE argues against transfer of $\mathrm{H}^{+}$being the rate-limiting step; however, it cannot exclude it. Possibly, reduction-associated distortion of FAD impeded by the protein environment is rate limiting for the reduction process.

Reoxidation of $\mathrm{FADH}_{2}$. The rate of reoxidation of $\mathrm{FADH}_{2}$ by oxygen in $L p P O X$ catalysis could be estimated from the concentration ratio of $[\mathrm{FAD}] /\left[\mathrm{FADH}_{2}\right]$ at steady-state and the measured rate constants of decarboxylation and reduction and the total of reoxidation and phosphorolysis (Figures $2-4)$. The pseudo-first-order rate constant of the reoxidation calculated in this way is about $k_{\text {reox }}=35 \pm 6 \mathrm{~s}^{-1}$. Under the experimental conditions, oxygen is not saturating $\left[K_{\mathrm{M}}\left(\mathrm{O}_{2}\right)\right.$ $\left.=300 \mu \mathrm{M},\left[\mathrm{O}_{2}\right]_{\exp }=211 \mu \mathrm{M}\right]$.

No flavin radicals could be observed under steady-state conditions as well as during electron transfer. A further question is whether the reduced flavin in $L p P O X$ reacts with dioxygen under formation of a covalent $4 \mathrm{a}$-hydroperoxide (28). During steady-state, spectral changes indicative for C4aadducts were not observed. We hope to clarify this point by studying the reoxidation reaction upon anaerobic reduction of the enzyme-bound flavin using stopped-flow/diode array spectrometry.

On the other hand, at steady-state and in the absence of phosphate, half-reduced FAD species were observed (Figure $9 \mathrm{~A}, \mathrm{~B})$, indicating that fully reduced FAD might be reoxidized stepwise under formation of $\mathrm{O}_{2}^{-} / \mathrm{HO}_{2}{ }^{-}$.

Phosphorolysis and Hydrolysis. The pseudo-first-order rate constant of phosphorolysis has been determined as $k_{\text {phos }}=$ $35 \pm 6 \mathrm{~s}^{-1}$. This value is in the same range as the $k_{\text {cat }}$ value determined in the artificial oxidation reaction using 2,6dichlorophenolindophenol and indicates that the phosphorolysis is partially rate-limiting in this artificial reaction. Studies conducted in the absence of phosphate point to a hydrolytic cleavage of the acetyl-ThDP intermediate proceeding with a rate constant of $k=0.03 \mathrm{~s}^{-1}$. Under comparable conditions $\left(\mathrm{pH} 6.0,24{ }^{\circ} \mathrm{C}\right)$, free acetyl-ThDP undergoes hydrolytic cleavage with a rate constant of $k=$ $0.2 \mathrm{~s}^{-1}(29,30)$, indicating that the enzyme-bound acetylThDP is shielded from the water by the enzyme environment.

There is strong evidence that free acetyl-ThDP undergoes neither adduct formation with phosphate, as in the case of the 2-acetyl-3,4-dimethylthiazolium ion (31), nor transfer of the acetyl group to phosphate.

Nevertheless, $L p \mathrm{POX}$ specifically favors phosphorolysis as opposed to hydrolysis. But the mechanism of phosphate activation is still unclear in molecular detail. The only base being sufficiently close to interact with phosphate is the carboxylate group of glutamate 483 , which could abstract one proton from $\mathrm{H}_{2} \mathrm{PO}_{4}{ }^{-}$to form the dianionic phosphate as a better nucleophile compared to the monoanionic form (Scheme 3). 
Scheme 3

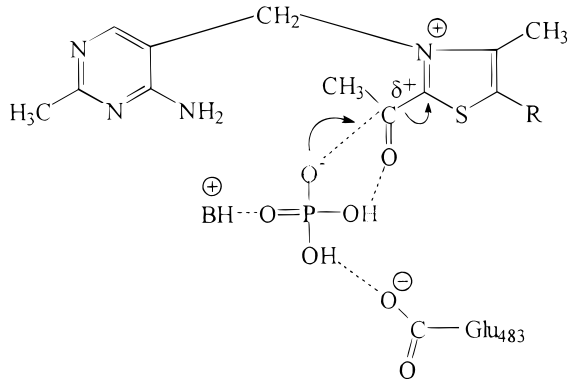

The observed kinetic solvent isotope effect in $k_{\text {cat }}$ of $k_{\mathrm{H}} / k_{\mathrm{D}} \approx 1.8$ and the linearity of the proton inventory curve indicate that a proton process involving one proton in the transition state is at least partial rate-limiting, or rate-limiting with an asymmetric transition state (32). This reaction influenced by the H/D isotope effect could be either flavin reoxidation or phosphorolysis.

\section{ACKNOWLEDGMENT}

We thank Michael Spinka and Georg Wille for helpful discussions.

\section{REFERENCES}

1. Sedewitz, B., Schleifer, K. H., and Götz, F. (1984) J. Bacteriol. 160, 273-278.

2. Sedewitz, B., Schleifer, K. H., and Götz, F. (1984) J. Bacteriol. $160,462-465$.

3. Götz, F., and Sedewitz, B. (1991) in Biochemistry and Physiology of Thiamin Diphosphate Enzymes (Bisswanger, H., and Ullrich, J., Eds.) pp 286-293, Verlag VCH Weinheim, Basel.

4. Muller, Y. A., and Schulz, G. E. (1993) Science 259, 965967.

5. Muller, Y. A., Schumacher, G., Rudolph, R., and Schulz, G. E. (1994) J. Mol. Biol. 237, 315-335.

6. Tittmann, K., Proske, D., Spinka, M., Ghisla, S., Rudolph, R., Hübner, G., and Kern, G. (1998) J. Biol. Chem. 273, 12929-12934.

7. Kern, D., Kern, G., Neef, H., Tittmann, K., Killenberg-Jabs, M., Wikner, C., Schneider, G., and Hübner, G. (1997) Science $275,67-70$.
8. Bertagnolli, B. L., and Hager, L. P. (1991) J. Biol. Chem. 266, 10168-10173.

9. Recny, M. A., and Hager, L. P. (1983) J. Biol. Chem. 258, 5189-5195.

10. Mather, M. W., and Gennis (1985) J. Biol. Chem. 260, $16148-$ 16155.

11. Gibson, Q. H., Swoboda, B. E. P., and Massey, V. (1964) J. Biol. Chem. 239, 3927-3934.

12. Kuzmic, P. (1996) Anal. Biochem. 237, 260-273.

13. Risse, B, Stempfer, G., Rudolph, R., Möllering H., and Jaenicke, R. (1992) Protein Sci. 1, 1699-1709.

14. Gadda, G., Wels, G., Pollegioni, L., Zucchelli, S., Ambrosius, D., Pilone, M. S., and Ghisla, S. (1997) Eur. J. Biochem. 250, 369-376.

15. Massey, V., Muller, F., Feldberg, R., Schuman, M., Sullivan, P. A., Howell, L. G., Mayhew, S. G. Matthews, R. G., and Foust, G. P. (1969) J. Biol. Chem. 244, 3999-4006.

16. Alvarez, F. J., Ermer, J., Hübner, G., Schellenberger, A., and Schowen, R. L. (1991) J. Am. Chem. Soc. 113, 8402-8409.

17. Risse, B. (1992) Thesis, Universität Regensburg.

18. Sun, S., Duggleby, R. G., and Schowen, R. L. (1995) J. Am. Chem. Soc. 117, 7317-7322.

19. Kluger, R., Chin, J., and Smyth, T. (1981) J. Am. Chem. Soc. $103,884-888$.

20. Crosby, J., Stone, R., and Lienhard, G. B. (1969) J. Am. Chem. Soc. 92, 2891-2900.

21. Crosby, J., and Lienhard, G. E. (1970) J. Am. Chem. Soc. 92, 5707-5716.

22. Kluger, R. (1987) Chem. Rev. 87, 863-876.

23. Pollegioni, L., Blodig, W., and Ghisla, S. (1997) J. Biol. Chem. 272, 4924-4934.

24. Chiu, C. C., Pan, K., and Jordan, F. (1995) J. Am. Chem. Soc. 117, 7027-7028.

25. Nakanishi, I., Itoh, S., Suenobu, T., Inoue, H., and Fukuzumi, S. (1997) Chem. Lett., 707-708.

26. Nakanishi, I., Itoh, S., Suenobu, T., Inoue, H., and Fukuzumi, S. (1997) Chem. Commun., 1927-1928.

27. Page, C. C., Moser, C. C., Chen, X., and Dutton, L. (1999) Nature 402, 47-52.

28. Ghisla, S., and Massey, V. (1989) Eur. J. Biochem. 181, 1-17.

29. Gruys, K. J., Halkides, C. J., and Frey, P. A. (1987) Biochemistry 26, 7575-7585.

30. Gruys, K. J., Datta, A., and Frey, P. A. (1989) Biochemistry 28, 9071-9080.

31. Lienhard, G. E. (1966) J. Am. Chem. Soc. 88, 5642-5649.

32. Gandour, R. D., and Schowen, R. L., Eds. (1978) Transition states of biochemical processes, Plenum Press, New York and London. 